\title{
Contents, Vol. 36, 1916
}

ínhalts -Verzeichnis.

Original-Arbeiten. Sette

Bachstetz, E., Zur Bewertimg der Sellaerweiterung im Röntgen-

bilde 62

Birch-Hirschfeld, A., Einige Bemerkungen zu den plastischen

Operationen an Lidern, Bindebaut und Orbita bei Schuß-

verletzungen. (Hierzu Tafel I-II) 36

Carsten, Paul,' Ein Fall von Cysticercus subretinalis. (Hierzu

Tafel III-IV) 203

-,- Über Ekblepharon-Prothesen 209

Goldmann, Rudolf, Die Hemeralopie als Teilerscheinung eines

zerebralen Symptomenkomplexes 220

v. d. Hoeve, Gesichtsfeld und Operationswahl bei Glaukom . 232 Hühn, K., Der Einfluß

fieberhafter Erkrankungen und Milch -

injektionen auf das Trachom und die Ophthalmogonorrhoe 305 Junius, P. Einige Bemerkungen

zur Nachtblindheit bei

Kriegsteilnehmern 49

Köhne, W., Umschriebene Grubenbildung im Bereich eines

Koloboms am Sehnerveneintritt. (Hierzu Tafel V.) . . 212 Koster, W., Eine kleine Änderung der Entropion-Operation, nach Carron du Villards

32

-,- Spontane Resorption eines Altersstars nebst einigen

Bemerkungen über anderweitige Naturheilungen am Auge 57 -,- Akute Linsentrübung mit schneller völliger Heilung

bei Glaucoma inflammatorium acutum

66

,-- Pseudo-Glaucoma simplex

193

Kraupa-Runk, M., Ein Beitrag zur Kenntnis kongenitaler

Anastomosen der Papillen- und Netzhautgefäße . . . 217 Kuhnt, H., Über plastische Operationen am Augapfel, an den

Lidern und der Orbita 1

-,- Über Neubildung von in ganzer Dicke und in ganzer

oder umschriebener Ausdehnung zu Verlust gekommenen

Augenlidern 147

-,- Schluß eines Hornhautdefektes durch Übertragung

von Fascia lata300

- IV -

Seite

Ohm, Jolh., Eine Registriervorrichtung für wagerechte Augen-

und Lidbewegungen 198 
-,- Das Ohrlabyrin,th als Erzeuger des Schielens. (Hierzu

Taf. VI-IX.) 253

Picl $\cdot$ L $\cdot r$, Alexius, Die Casparsche Ringtrübung der Hornhaut 311 Reis, W., Zur Beurteilung der Einbuße an Erwerbsfähigkeit

infolge traumatischer homonymer Hemianopsie 273

Schav $>$ z, Fritz, Lichtbehandlung bei Augenleiden ..... 22

Steiger, Adolf, Streiflichter zur Myopiefrage 127

Stoewer, P., Thrombophlebitis mit Verlust eines Auges nach

Tränensackexstirpation $\quad 310$

-,- Netzhautablösung und Augenmuskellähmung bei

Schwangerschaftsnephritis 314

Berichte über die ausländische ophthalmologische Literatur.

Französische Literatur 1916 (I. Semester). Referate. Von

Dr. Pflüger in Bern 112

Holländische ophthalmologische Literatur des Jahres 1916(1. Hälfte). Von Prof. Dr. W. Koster-

Gzn in Leiden undDr. G. J. Schoute in Amsterdam 231

Gesellschaftsberichte.

Rericht über die XI. (außerordentliche) Zusammenkunft der Ophthalmologischen Gesellschaft in Heidelberg am 31. VII.

a. 1. VIII. 1916. Von Prof. Star gar dt in Bonn ...

Tberapeutische Umsehau 243, 322

Unfall- und Versicherungskunde 120,246

Bücherbesprechungen 125, 335

Literaturverzeichnis 121, 248, 343

Personalien $\quad 126$

Druckfehler-Berichtigung

S. Sachregister $\quad 347$

Namenregister $\quad 351$ 